\title{
El reflujo gastroesofágico sintomático se asocia fuertemente con el adeno- carcinoma esofágico
}

\author{
Symptomatic gastroesophageal reflux as a risk factor for esophageal adenocarinoma. Lagergren J, Bergström R, Lindgren A et al. $\mathrm{N}$ Engl $\mathrm{J}$ \\ Med 1999;340:825-831
}

\section{Objetivo}

Estimar la asociación del reflujo gastroesofágico (RGE) sintomático con el adenocarcinoma de esófago y de cardias.

\section{Diseño}

Estudio de caso-control* poblacional realizado entre los años 19951997.

\section{Lugar}

Estudio Nacional en Suecia.

\section{Pacientes}

De toda la población sueca menor de 80 años en el período referido se compararon 189 pacientes con adenocarcinoma de esófago y 262 pacientes con adenocarcinoma de cardias con 167 pacientes con carcinoma esofágico epidermoide y 820 controles.

\section{Evaluación de los factores de riesgo}

Los participantes respondieron a una encuesta acerca del reflujo gastroesofágico de entre 169 y 553 preguntas.

\section{Medición de resultados principales}

Fueron enrolados todos los casos de adenocarcinoma de esófago y cardias diagnosticados entre 1994 y 1997. El efectivo reclutamiento se llevo a cabo a través de una organización que involucraba contac- tos personales con profesionales de 195 departamentos quirúrgicos y la colaboración continua de registros de tumores de 6 regiones. Los controles fueron seleccionados en forma aleatoria a través de un registro poblacional.

\section{Resultados principales}

El reflujo frecuente ( 1 vez por semana) y el reflujo nocturno periódi$\mathrm{co}$, tuvieron respectivamente un riesgo 8 y 11 veces más elevado que la población control de padecer adenocarcinoma de esófago. Al realizar el análisis multivariable, * ajustando por el efecto de la edad, sexo, índice de masa corporal, tabaco, alcohol, dieta y nivel socio-económico; los pacientes con RGE más de 3 veces por semana tuvieron un Odds Ratio* de 17 (IC95\% 8,7-28,3) para adenocarcinoma de esófago. Cuando el puntaje de severidad de RGE fue mayor de 4,5 el 0 R fue de 20 (IC95\% 11,6-34,6) y cuando la duración había sido mayor de 20 años el OR fue de 17 (IC95\% 8,3-28,4). La asociación entre la frecuencia y severidad del RGE con el cáncer de cardias fue: OR 2,3 (IC95\% 1,2-4,8) para RGE $3 \times$ semana; OR 2,8 (IC95\% 1,6-5) para el de puntaje alto y 3,3 (IC95\% 1,8-6,8) para el de 20 años de duración. No hubo asociación alguna con el carcinoma epidermoideo de esófago.

\section{Conclusiones}

Hay una asociación muy fuerte entre el RGE y el adenocarcinoma de esófago, y una asociación más débil con el adenocarcinoma de cardias.

\section{COMENTARIO}

La incidencia de adenocarcinoma de esófago y cáncer de cardias han aumentado en 0 ccidente en las últimas décadas ${ }^{1}$, siendo más pronunciada para el primero de estos tumores. Sin embargo las razones del aumento de dicha incidencia son desconocidas.

Este trabajo muestra claramente una fuerte asociación del RGE sintomático con el adenocarcinoma de esófago y una asociación más débil con el cáncer de cardias. La fuerza del estudio esta dada en la gran muestra poblacional considerada, la clasificación uniforme de los tumores y el completo y rápido reclutamiento de los casos. La entrevista realizada por encuestadoras profesionales recabaron datos sobre síntomas típicos de reflujo (pirosis y/o regurgitación) que habían comenzado 5 o más años previos al diagnóstico de cáncer para poder evaluar adecuadamente la temporalidad de la asociación Aunque las encuestadoras no estaban ciegas en relación con los casos, sí lo estaban, al igual que los pacientes, a la estirpe histológica de los tumores. Por esta razón es poco probable que existan sesgos de entrevista o recuerdo diferentes para cada tumor. Los potenciales factores confundidores fueron identificados y controlados en el análisis (regresión logística múltiple). La tasa de rechazo en participar entre los controles fue del $27 \%$. Esto podría haber introducido un sesgo, sin embargo es improbable que la no participación de éstos este ligada selectivamen-

\section{*Ver glosario}

\section{Dr. Jorge A. Olmos}

Servicio de gastroenterología. Hospital Italiano de Buenos Aires. te al RGE.

Es llamativo el hecho de que sólo en el $62 \%$ de los adenocarcinomas de esófago se detectara esófago de Barrett, no hallándose diferencias en la fuerza de asociación con el RGE entre los que tenían y los que no tenían metaplasia esofágica.?

Los principios de causalidad están presentes 1) La temporalidad (los sintomas de RGE preceden al tumor); 2) La notable fuerza de la asociación; 3) La consistencia (esto ya fue sugerido en un estudio previo); 4) el gradiente dosis respuesta (a mayor severidad de reflujo o mayor duración, mayor riesgo); y 5) La plausibilidad biológica.

Desde el punto de vista clínico está acordado el seguimiento endoscópico con biopsias de los pacientes con esófago de Barrett con el objetivo de encontrar displasia o tumores tempranos ${ }^{3}$. Sin embargo los pacientes con RGE que no tienen Barrett y que son el grupo mayoritario no son factibles de seguimiento. En nuestro país el reflujo frecuente lo tiene el $23 \%$ de la población (datos no publicados). Por otro lado en Suecia habría que seguir más de 1400 pacientes para encontrar un caso lo cual no sería costo efectivo.

En resumen, parece claro la asociación de reflujo severo y duradero con el adenocarcinoma y tal vez con el cáncer de cardias; resta demostrar si el tratamiento del RGE puede prevenir la aparición del mismo (criterio último y definitivo de causalidad).

\section{Referencias}

1. Pera M, Cameron AJ, Trastek VF et al. Increasing incidence of adenocarcinoma of the esophagus and esophagogastric junction. Gastroenterology 1993; 104: 510-3.

2. Chow WH, Finkle WD, McLaughtin JK, et al. The relation of gastroesophageal reflux disease and treatment to adenocarcinomas of the esophagus and gastric cardia. JAMA 1995; 274: 474-7 3. Streitz JM Jr, Ellis FH Jr, Tilden RL, et al. Endoscopic surveillance of Barrett's esophagus: a cost-effectiveness comparison with mammographic for breast cancer. Am J Gastroenterol 1998; 93:911-5 